\title{
Relação entre variáveis meteorológicas e a qualidade industrial do trigo
}

\author{
Relation between meteorological variables and the industrial quality of the wheat
}

\author{
Simone Aparecida Miloca ${ }^{\text {I }}$ Anselmo Chaves Neto ${ }^{\text {II }}$ Neida Maria Patias Volpi ${ }^{\text {II }}$ \\ Paulo Domingos Conejo ${ }^{\mathrm{IV}}$
}

\section{RESUMO}

O trigo é um cereal utilizado na fabricação de inúmeros produtos e as indústrias, ao efetuarem a compra desse cereal, observam alguns parâmetros de qualidade industrial, que usualmente são determinados através de testes como peso hectolitro $(\mathrm{PH})$, força de glúten, número de queda, dentre outros, realizados em laboratórios especializados. No Brasil, os maiores produtores de trigo são os Estados do Rio Grande do Sul, do Paraná e de Santa Catarina, onde a variabilidade climática é grande, o que pode ser um fator de influência nos resultados dos testes de qualidade. O objetivo deste trabalho é fazer uma avaliação estatística de algumas variáveis meteorológicas que afetaram a qualidade industrial do trigo em algumas regiões do Estado do Paraná nos anos de 1999, 2000 e 2001, utilizando técnicas da análise estatística multivariada. Assim, ajustou-se um modelo de regressão linear multivariada para se tentar explicar o relacionamento do conjunto de variáveis respostas IQI (da qualidade) com o conjunto de variáveis explicativas IQP. Da mesma forma, estimou-se a correlação canônica entre estes dois conjuntos de variáveis, que expressa o relacionamento entre os dois conjuntos. Os modelos de regressão multivariada desenvolvidos não se mostraram suficientemente eficientes para prever a qualidade em função das variáveis climáticas, porém as correlações canônicas estimadas provaram a existência do relacionamento entre as variáveis de qualidade (IQI) e as variáveis climáticas (IQP).

Palavras-chave: clima, regressão multivariada, correlação canônica.

\section{ABSTRACT}

The wheat is a product used in the manufacture of several products. The industries, when effecting the purchase of this cereal, observe some parameters of industrial quality, that can be determined through some tests (weight hectolitro $(P H)$, gluten strength, Falling Number), carried out in specialized laboratories. In Brazil, the main producers of wheat are the states of Rio Grande do Sul, Paraná and Santa Catarina, and in these states, the climatic variability is great and this can be a factor of influence in the results of the quality tests. This work research is aimed at making a statistics evaluation, of some meteorological variables that affected the industrial quality of the wheat in some regions of the state of Parana in the years 1999, 2000 and 2001. This evaluation will be carried out through techniques of the multivaried analysis. Thus, a model of linear regression multivaried was adjusted to try itself to explain the relationship of the set of changeable answers IQI (of the quality) with the set of explicativas variables IQP. In the same way it was esteem canonic correlation enters these two sets of variables, that express the relationship between the two sets. The models of multivaried regression developed weren't enough efficient to predict the quality concerning the climatic variable, however the canonic correlations estimates proved the existence of the relationship between the variables (IQI) of quality and the climatic variables(IQP).

Key words: climate, multivariate analysis, canonical correlation.

\section{INTRODUÇÃO}

De acordo com a FAO (Food and Agriculture Organization), no mundo todo, o trigo é apontado como a segunda cultura de grãos em produção, sendo o primeiro lugar ocupado pelo milho. De acordo com estimativas da CONAB (Conselho

\footnotetext{
ICentro de Ciências Exatas, Universidade Estadual do Oeste do Paraná (Unioeste), Rua Universitária, 2069, CP 000711, 85819110, Cascavel, PR, Brasil. E-mail: smiloca@unioeste.br. Autor para correspondência.

IIDepartamento de Estatística, Universidade Federal do Paraná (UFPR), Curitiba, PR, Brasil. E-mail: anselmo@est.ufpr.br.

IIIDepartamento de Matemática, UFPR, Curitiba, PR, Brasil. E-mail: nmpv@mat.ufpr.br.

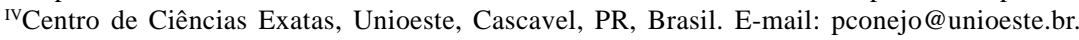


Nacional do Abastecimento), o Brasil produz entre 2 e 5 milhões de toneladas por ano para um consumo de mais ou menos 10 milhões de toneladas. Entidades de pesquisa e segmentos interessados se esforçam no sentido de obter um crescimento na produção.

O trigo, geralmente, é plantado em cada região segundo recomendações feitas por órgãos de pesquisa como o IAPAR $(1999,2000,2001)$ e a EMBRAPA Trigo. Os maiores produtores deste grão no Brasil são os Estados do Paraná, do Rio Grande do Sul e de Santa Catarina, com aproximadamente $90 \%$ da produção brasileira.

Após a colheita, o trigo é enviado aos moinhos, onde é transformado em um produto farináceo ou granular (farinha ou semolina), sendo a indústria do trigo responsável pela fabricação de inúmeros produtos, em sua grande maioria utilizados na alimentação humana. Ao se avaliar o destino do trigo, um fator de grande importância é sua qualidade industrial, pois, dependendo de sua classificação, a mesma farinha pode ser boa para fabricar determinados produtos e não ser indicada para outros. Esta classificação é feita através de vários testes físicoquímicos e reológicos, realizados em laboratórios especializados (GUARIENTI, 1993; 2001).

Estudos mostram que os principais fatores que influenciam os resultados dos testes de qualidade industrial do trigo são: i) a genética (cultivar) da semente: muitos estudos e pesquisas foram feitos por órgãos de pesquisa, tais como a COODETEC (Cooperativa Central de Pesquisa Agrícola), e pela EMBRAPA Trigo, para a melhoria das sementes plantadas em cada região do sul do país; a semente plantada pode indicar a aptidão do trigo/farinha para a fabricação de diferentes produtos tais como pães, biscoitos, bolachas, massas, etc.; ii) a fertilização do solo: após efetuar-se a análise do solo, é possível se estimar a necessidade de calcário e fertilizantes; uma boa fertilidade do solo colabora com a sanidade da planta e, conseqüentemente, do grão, e, se mantida até a colheita, ajuda no processo de qualificação do trigo; iii) o clima da região: dependendo do clima (desde o plantio até a colheita), a genética e os tratamentos que foram aplicados à cultura no campo podem levar a um bom resultado ou a um mau resultado, principalmente quanto à qualidade do grão; iv) a secagem dos grãos: esta deve ser feita de forma correta a fim de garantir a qualidade intrínseca do grão, pois, se for feita incorretamente, destruirá principalmente a qualidade do glúten e do amido; v) a armazenagem: esta manterá ou prejudicará todas as qualidades do grão; assim, se realizada de forma correta, poderá até melhorar as qualidades reológicas (BRUNETTA et al., 1997).
Técnicos especializados da área relatam que a fertilização, a secagem e a armazenagem são fatores que, no período do plantio até a colheita e a póscolheita, podem ser bem administrados, sendo a maior preocupação os fatores relacionados ao clima e à genética. Nos Estados do Paraná, de Santa Catarina e do Rio Grande do Sul, a variabilidade climática é muito grande, fazendo com que a produção tritícola seja uma atividade de risco. Publicações sobre este assunto podem ser encontradas em BRUNETTA et al. (1997), GUARIENTI (1993; 2001), IAPAR (1999; 2000; 2001)e na Reunião da Comissão Sul Brasileira de trigo (1999, 2001).

O objetivo deste trabalho é fazer um estudo do relacionamento entre o vetor de variáveis meteorológicas e o vetor de índices de qualidade industrial, em determinadas regiões do Estado do Paraná, com observações de um período determinado. Devido à estrutura de dados multivariados, para se atingir tal objetivo, a idéia é utilizar-se técnicas da análise multivariada.

\section{MATERIAL E MÉTODOS}

As observações amostrais foram fornecidas pela COAMO - Cooperativa Agrícola de Campo Mourão, e se referem às colheitas de trigo dos períodos de 1999, 2000 e 2001. Esses dados correspondem às colheitas nas cidades onde a Cooperativa possui entreposto de recebimento e armazenamento de trigo no Estado do Paraná, ou seja, Ivaiporã, Fênix, Quinta do Sol, São João, Luiziana, Farol, Boa Esperança, Mamborê, Juranda, Tupassi, Bragantina, São Pedro do Iguaçu, Vila Nova, Iretama, Roncador, Pitanga, Coronel Vivida, Mangueirinha e, em Santa Catarina, na cidade de Ouro Verde (próxima à divisa com o Estado do Paraná).

A COAMO forneceu os dados que coletou em cada localidade e que correspondem a um número de amostras variando entre 4 e 15 em cada localidade. Dessa forma, teve-se disponibilidade de 139 amostras no ano de 1999, de 69 no ano de 2000 e de 93 no ano de 2001. O processo de obtenção de tais amostras foi o seguinte. No período de colheita, os produtores entregam o trigo na cooperativa ou em entrepostos e, no momento do recebimento, uma amostra dos grãos é formada por várias subamostras retiradas de pontos aleatórios do caminhão que o transporta. A partir das informações dessa amostra, é feita uma classificação do trigo quanto ao tipo, segundo instrução normativa no 1, de 27 de janeiro de 1999, do MAA (Ministério da Agricultura e Abastecimento). Essa instrução estabelece normas de identidade e qualidade do trigo.

Ciência Rural, v.37, n.1, jan-fev, 2007. 
Dependendo do tipo, o trigo é separado para o envio ao processo de secagem e armazenamento. Antes do processo de secagem, é feita uma eliminação das impurezas. Logo após isso, o trigo é guardado em um armazém que possui várias células, com capacidades diferenciadas de armazenamento.

No final do processo de secagem dos grãos, a cada período de tempo, usualmente $20 \mathrm{~min}$, procedese a coletas de trigo até que se obtenha uma meia saca. Esta meia saca constitui uma amostra. Essa amostra é, então, enviada ao laboratório para que se façam as análises. De cada amostra (meia saca) é extraída a farinha, em um simulador de moinho, e, com essa farinha, são feitas as análises. As análises fornecerão indicadores que definem a qualidade industrial. Os principais indicadores se referem ao Glúten Úmido (GU), ao Glúten Seco (GS), ao Falling Number (FN), a Estabilidade (EST), à relação P/L (pressão máxima de ruptura/extensibilidade), à Força de Glúten (W) e à Absorção de Água (AA). Tais indicadores constituem o conjunto de variáveis aleatórias da qualidade, ou seja, o vetor aleatório das variáveis de qualidade, denotadas neste trabalho por IQI. Essas variáveis são contínuas, pois o resultado de cada análise é expresso por um número real. Uma descrição de tais análises pode ser encontrada em GONZALEZ \& LOPES (1992), BRUNETTA et al. (1997) e GUARIENTI \& MIRANDA (1999).

O número de amostras obtidas em cada entreposto é, geralmente, maior do que um. Então, calcula-se a média amostral que representará o valor final considerado para cada análise. Conseqüentemente, tem-se uma matriz de ordem 19 x 7 (19 entrepostos e 7 variáveis).

Vale notar que o trigo não é separado pelo tipo de cultivar, mas sim pelo tipo (1, 2 ou 3 , conforme consta na instrução normativa $\mathrm{n}^{-0} 1$ do MAA). Isto ocorre por diversos motivos, entre os quais, o fato de o produtor ter colocado no caminhão diversas cultivares, ou ainda, de exigir uma quantidade grande de células nos armazéns de estocagem. Cabe ressaltar, ainda, que os resultados das análises foram obtidos no final do processo de secagem, sendo este o trigo a ser enviado aos moinhos e posteriormente utilizado na fabricação dos mais diversos produtos.

Quanto aos dados climáticos, considerouse: a temperatura mínima no período de pré-colheita (Tmin, em ${ }^{\circ} \mathrm{C}$ ); a temperatura máxima no período de précolheita (Tmax, em ${ }^{\circ} \mathrm{C}$ ); a umidade (Umid, em \%) e a precipitação (Precip, em mm). O conjunto dessas variáveis aleatórias foi denominado por IQP e constitui o vetor aleatório das variáveis climáticas. As componentes desse vetor foram medidas nas estações climáticas da cooperativa nas regiões onde ficam localizados os armazéns. O clima considerado foi o da pré-colheita. Segundo recomendações feitas por técnicos da área de trigo, para a região norte, noroeste, centro-oeste e oeste, os meses indicados são agosto, setembro e outubro. Para a região centro, os meses indicados são setembro, outubro e novembro e, para a região centro-sul e sul, outubro, novembro e dezembro. Com relação a cada amostra, considerou-se para as variáveis Tmin, Tmax e Umid., a média aritmética simples dos valores medidos no mês considerado como o da pré-colheita na região em questão. $\mathrm{O}$ valor final, tomado para cada entreposto, de cada uma destas variáveis foi a média aritmética dessas médias. Para o índice de precipitação, considerou-se o somatório dos valores obtidos no mês considerado como pré-colheita. Observe-se que se tem para os dados IQP uma matriz de ordem 19 x 4 (19 entrepostos e 4 variáveis climáticas).

A metodologia aplicada foi aquela multivariada, própria para obtenção de um modelo que relacione o vetor IQI das $q=7$ variáveis aleatórias de qualidade com as $p=4$ variáveis aleatórias meteorológicas. Na forma matricial, o modelo pode ser escrito como:

$$
Y_{n \times q}=X_{n \times p} B_{p \times q}+\varepsilon_{n \times q}
$$

em que, $Y$ é a matriz de ordem $n \times q$ das $n$ observações nas $q$ variáveis respostas (dependentes); $X$ é a matriz do modelo de ordem $n \times p$ das $n$ observações das $p$ variáveis regressoras (independentes); $B$ é a matriz de ordem $p \times q$ dos coeficientes de regressão; $\varepsilon$ é a matriz de ordem $n \times q$ dos erros. Assim, cada vetor $\underline{\mathrm{Y}}$, da matriz $\mathrm{Y}$, tem o seu próprio modelo de regressão linear univariado.

Em geral, admite-se que cada coluna da matriz dos erros $\varepsilon$ tenha distribuição aproximadamente normal com média zero e variância $\sigma^{2}$. É claro que esta suposição deve ser verificada antes da realização de inferências estatísticas com o ajuste do modelo.

Já um modelo de regressão linear univariado e polinomial, de forma geral, é dado por

$$
y=a_{0}+a_{1} x+a_{2} x^{2}+\ldots+a_{k} x^{k}+\varepsilon
$$

Para a realização de inferências válidas sobre os parâmetros de regressão, efetua-se o teste da existência de regressão (ANOVA -univariado e MANOVA-multivariado), que tem o objetivo de verificar a possibilidade de os parâmetros do modelo serem todos nulos (exceto o intercepto) e , neste caso, não existe regressão (BARROS NETO et al.,1995). Para tais inferências, o teste de Bartlett (KOEHLER,1998) foi feito no sentido de verificar se existe ou não diferença significativa entre as variâncias das amostras analisadas, associadas às variáveis respostas, 
provenientes das diferentes cidades. Além disso, o teste de Kolmogorov-Smirnov, também foi realizado para verificar a normalidade da distribuição das amostras. Maiores detalhes desses testes podem ser obtidos em JOHNSON \& WICHERN (1988), CHAVES NETO(1998) e MOOD \& GRAYBILL(1986). Aqualidade do modelo escolhido para o ajuste dos dados é avaliada pelo coeficiente de determinação $\mathrm{R}^{2}$. Quanto mais próximo de 1 estiver essa estatística, melhor será o ajuste. Se $\mathrm{R}^{2}$ é próximo de zero, o modelo não é adequado.

A matriz de correlação das variáveis explicativas (independentes) envolvidas no modelo foi estimada e, então, excluiu-se da matriz de dados as variáveis com alta correlação. Dessa forma, foi possível selecionar as variáveis que fizeram parte do modelo de regressão linear, evitando-se o problema da multicolinearidade. O teste de autocorrelação de Durbin-Watson foi aplicado e comprovou a utilidade do procedimento, uma vez que existia multicolinearidade.

A intensidade do relacionamento entre os vetores IQP e IQI foi avaliada por meio da análise de correlação canônica. Como é bem sabido, o objetivo principal da correlação canônica é obter pares de variáveis aleatórias que sejam combinações lineares das variáveis de um conjunto, o vetor $\underline{X}$, e de um outro conjunto, o vetor $\underline{Y}$, de modo que tenham a mais alta correlação entre si. Os pares de combinações lineares são chamados pares de variáveis canônicas e suas correlações são as correlações canônicas. Portanto, a correlação canônica mede o grau de associação entre dois conjuntos de variáveis.

\section{RESULTADOS E DISCUSSÃO}

Inicialmente, os dados coletados foram armazenados em planilhas do Microsoft Excel, que posteriormente foram transportados para planilhas do software Statgraphics, onde os resultados foram obtidos.

O primeiro dos testes realizados foi o teste de Bartlett para a verificação da homogeneidade das variâncias das variáveis respostas. A seguir, aplicouse o teste de Kolmogorov-Smirnov para a verificação da normalidade. Como resultado, obteve-se, para o ano de 2001, que as variáveis PL e GS são heterogêneas e, em 1999 e 2000, que a variável PL é heterogênea. Foi aplicada aos dados destas variáveis a transformação logarítmica. Isto permitiu homogeneizar as variâncias. A escolha desta transformação se deve ao fato desta ser indicada nos casos em que existe uma certa proporcionalidade entre a média e o desvio padrão dos dados, conforme foi possível constatar. De acordo com o teste de Kolmogorov-Smirnov, obteve-se normalidade para todas as variáveis dependentes (respostas) em todos os anos avaliados.

Antes de se efetuar a MANOVA, calculouse a matriz de correlação das variáveis envolvidas. Para os dados de 1999, obteve-se alta correlação entre as variáveis GU e W (r=0,72), EST e AA (r=-0,77) e Tmin e Tmax ( $\mathrm{r}=0,61)$. Portanto, no modelo de regressão linear univariado, não foram consideradas as variáveis GU, EST e Tmin. Para o ano de 2000, a matriz de correlação indicou existência de alta correlação entre GU e GS $(\mathrm{r}=0,91)$, Tmax e GU $(\mathrm{r}=-0,60)$, Tmin e Tmax $(\mathrm{r}=0,56)$, Precip e Umid (r=-0,59). Assim, no modelo de regressão, não foram consideradas as variáveis GU, Umid e Tmin. Com relação ao ano de 2001, obteve-se as correlações entre: GS e EST ( $r=0,74)$, GS e GU ( $r=0,66)$, GS e FN $(\mathrm{r}=0,60)$, GS e W $(\mathrm{r}=0,67)$, GU e EST $(\mathrm{r}=0,52)$, Tmin e Precip ( $\mathrm{r}=-0,61)$ e Tmax e Precip $(\mathrm{r}=-0,64)$. Assim, retirando-se as variáveis GS, EST e Precip, obteve-se uma matriz de correlação, de modo que as demais variáveis não estivessem correlacionadas.

Os coeficientes para o modelo de regressão linear multivariada estão apresentados na tabela 1. A MANOVA foi feita no sentido de determinar se uma determinada variável independente tem relacionamento significativo com o grupo de variáveis dependentes. Com respeito ao ano de 1999, 2000 e 2001, observase que devido ao fato de o valor-p estar acima de 0,10 , nenhuma das variáveis independentes é estatisticamente significativa para a composição do modelo. Portanto, deve-se rejeitar o modelo linear proposto com um nível de significância $\alpha=0,10$.

Na tabela 1, apresentam-se também os resultados obtidos na análise de correlação canônica, bem como o primeiro par de variáveis canônicas. Os valores são 0,77 para 1999; 0,69 para 2000 e 0,77 para 2001. Os valores das correlações canônicas mostram que existe um relacionamento, no mínimo moderado, entre os conjuntos IQI e IQP. Portanto, o relacionamento entre os vetores IQP e IQI está comprovado. Porém, há necessidade de se estabelecer a forma do relacionamento (modelo).

Observa-se até o momento que, através das análises efetuadas, o modelo de regressão linear não é adequado para explicar o relacionamento entre as variáveis IQI e IQP. Uma tentativa de encontrar um modelo que melhor se ajuste aos dados foi feita utilizando-se um modelo quadrático. Os resultados são apresentados na tabela 2. Nota-se um ajuste significativo, de melhor qualidade do que o linear para os dados do ano de 1999. O teste $F$ da ANOVA confirma 
Tabela 1 - Resultados da regressão linear multivariada e da correlação canônica.

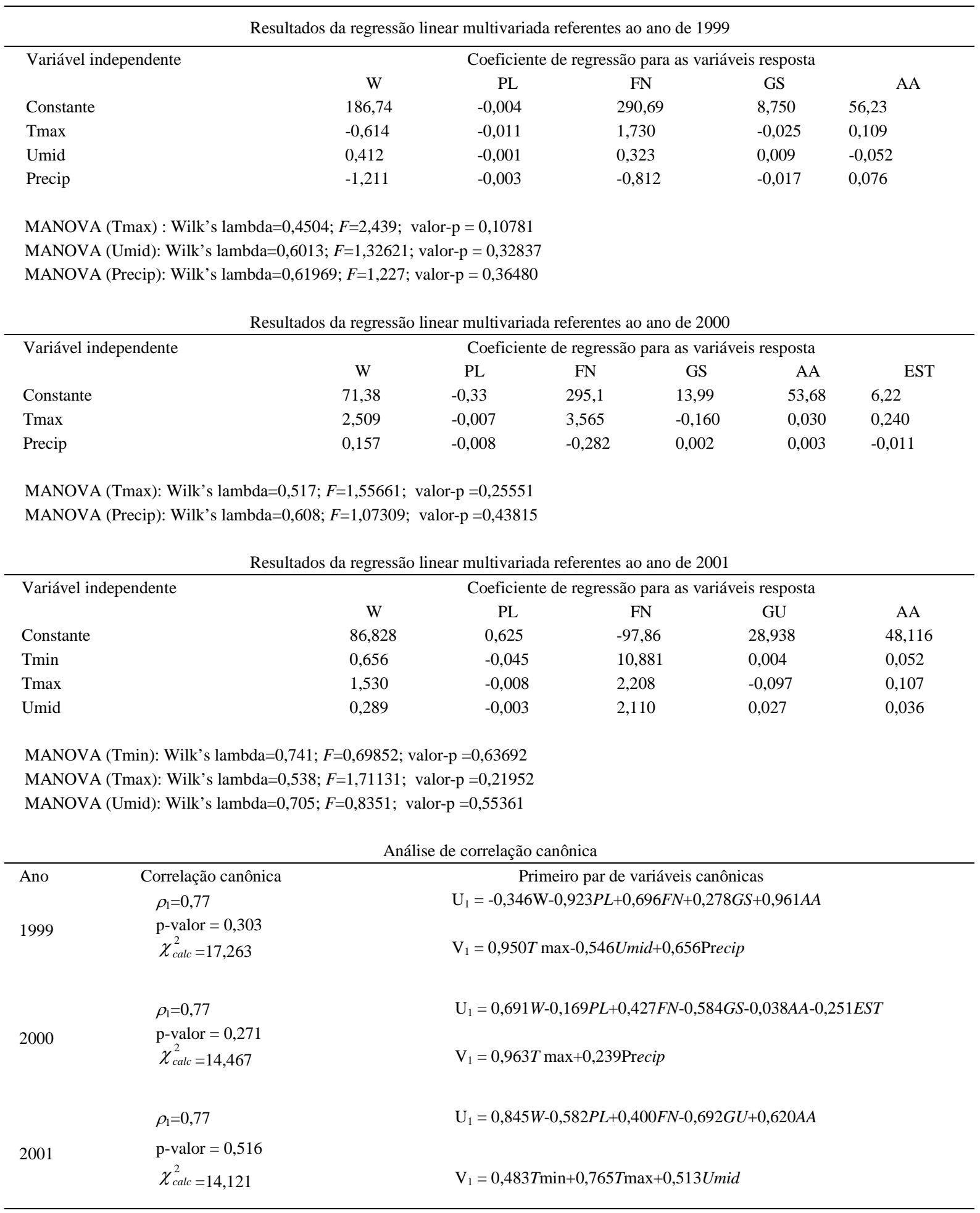

tal melhoria, pois o valor de $F_{\text {calc }}>F_{\text {tab }}$. A porcentagem da variação explicada $R^{2}$ indica que o modelo quadrático explica aproximadamente $58 \%$ da variação total de W, $63 \%$ da variação total em PL, 49\% da variação total em
FN, 31\% da variação total em GS e 74\% da variação total em AA.

No ajuste quadrático dos anos de 2000 e 2001, o valor de $F_{\text {calc }}$ da ANOVA indica um resultado

Ciência Rural, v.37, n.1, jan-fev, 2007. 
Tabela 2 - Coeficientes do ajuste de um modelo quadrático, valor de F obtido da ANOVA e valor de $R^{2}$.

\begin{tabular}{|c|c|c|c|c|c|c|c|c|c|c|c|c|}
\hline \multicolumn{13}{|c|}{ Com relação aos dados de 1999} \\
\hline$\hat{Y}$ & $a_{0}$ & $a_{1}$ & $a_{2}$ & $a_{3}$ & $a_{4}$ & $a_{5}$ & $a_{6}$ & $a_{7}$ & $a_{8}$ & $a_{9}$ & $F_{\text {calc }}$ & $R^{2}$ \\
\hline $\mathrm{W}$ & 383,9 & $-29,32$ & 6,15 & $-23,53$ & 0,68 & $-0,01$ & $-0,03$ & $-0,13$ & 0,54 & 0,12 & 417,8 & 58,82 \\
\hline PL & $-8,66$ & 0,34 & 0,12 & $-0,24$ & $-0,004$ & $-0,0006$ & $-0,0009$ & $-0,001$ & 0,002 & 0,002 & 15,71 & 63,64 \\
\hline FN & 49,38 & $-11,19$ & 11,84 & 56,43 & 0,64 & 0,003 & 0,043 & $-0,36$ & $-0,33$ & $-0,70$ & 205,11 & 48,38 \\
\hline GS & 3,28 & $-0,06$ & 0,22 & $-0,97$ & 0,004 & $-0,001$ & $-0,019$ & $-0,003$ & 0,007 & 0,015 & 328,03 & 31,93 \\
\hline AA & $-20,95$ & 4,40 & 0,65 & $-2,44$ & $-0,07$ & $-0,005$ & $-0,03$ & $-0,005$ & 0,003 & 0,041 & 5288 & 73,74 \\
\hline \multicolumn{13}{|c|}{$\hat{y}=a_{0}+a_{1} T \max +a_{2} U m i d+a_{3}$ Precip $+a_{4} T \max ^{2}+a_{5} U m i{ }^{7}+a_{6}$ Precip ${ }^{2}+a_{7} T \max T$ min $+a_{8} T \max$ Precip $+a_{9} U m i d$ Precip } \\
\hline \multicolumn{13}{|c|}{$F_{t a b}(0,10 ; 10,18)=2,54$} \\
\hline \multicolumn{13}{|c|}{ Com relação aos dados de 2000} \\
\hline$\hat{Y}$ & \multicolumn{2}{|c|}{$a_{0}$} & $a_{1}$ & \multicolumn{2}{|l|}{$a_{2}$} & $a_{3}$ & \multicolumn{2}{|l|}{$a_{4}$} & $a_{5}$ & $F_{\text {calc }}$ & \multicolumn{2}{|c|}{$R^{2}$} \\
\hline W & \multicolumn{2}{|c|}{$-892,77$} & 15,43 & \multicolumn{2}{|c|}{6,46} & $-0,12$ & \multicolumn{2}{|c|}{$-0,01$} & $-0,02$ & 1475,5 & \multicolumn{2}{|c|}{37,64} \\
\hline PL & \multicolumn{2}{|c|}{15,53} & $-0,44$ & \multicolumn{2}{|c|}{$-0,081$} & 0,008 & \multicolumn{2}{|c|}{0,0001} & $-0,0001$ & 24,22 & \multicolumn{2}{|c|}{1,76} \\
\hline FN & \multicolumn{2}{|c|}{3725,89} & $-193,16$ & \multicolumn{2}{|c|}{$-6,63$} & 3,03 & \multicolumn{2}{|c|}{0,004} & 0,14 & 92,38 & \multicolumn{2}{|c|}{5,42} \\
\hline GS & \multicolumn{2}{|c|}{$-1,87$} & 0,13 & \multicolumn{2}{|l|}{0,09} & 0,004 & \multicolumn{2}{|c|}{$-0,00007$} & $-0,002$ & 346,72 & \multicolumn{2}{|c|}{25,36} \\
\hline AA & & & $-1,55$ & $-0,29$ & & 0,01 & 0,0003 & & 0,003 & 6818,4 & 1,8 & \\
\hline EST & 56 & & $-8,05$ & 0,45 & & 0,217 & $-0,000$ & & $-0,01$ & 32,89 & 5,7 & \\
\hline$\hat{y}=a$ & $a_{1} T$ maxt & ${ }_{2}$ Precip- & $a_{3} T \max ^{2}$ & $a_{4}$ Precip ${ }^{2}$ & $+a_{5} T \mathrm{~m}$ & x.Precip - & $F_{\text {tab }}(0,10$; & $6 ; 12)=2$ & 2,33 & & & \\
\hline & & & & & Com re & ação aos dad & os de 2001 & & & & & \\
\hline$\hat{Y}$ & $a_{0}$ & $a_{1}$ & $a_{2}$ & $a_{3}$ & $a_{4}$ & $a_{5}$ & $a_{6}$ & $a_{7}$ & $a_{8}$ & $a_{9}$ & $F_{\text {calc }}$ & $R^{2}$ \\
\hline $\mathrm{W}$ & 768,5 & 221,8 & 62,82 & 74,82 & $-5,71$ & $-1,11$ & 0,32 & $-3,65$ & 0,46 & 0,61 & 69,04 & 4,87 \\
\hline PL & 82,28 & 4,47 & $-0,77$ & $-2,60$ & $-0,13$ & $-0,10$ & 0,01 & 0,22 & $-0,07$ & 0,04 & 7,26 & 6,89 \\
\hline FN & -9108 & $-216,2$ & $-14,18$ & 270,2 & 14,84 & 14,82 & $-1,63$ & -31 & 7,40 & $-4,01$ & 37,41 & 15,97 \\
\hline GU & $-343,6$ & $-6,89$ & 5,83 & 8,59 & 0,48 & 0,55 & $-0,05$ & $-1,47$ & 0,38 & $-0,17$ & 459,58 & 3,80 \\
\hline AA & 5,13 & 19,22 & $-4,05$ & $-0,97$ & $-0,42$ & 0,04 & 0,007 & $-0,05$ & $-0,07$ & 0,03 & 4616 & 15,29 \\
\hline$\hat{y}=a$ & $a_{1} T \min$ & $l_{2} T$ max + & $a_{3} U m i d+$ & ${ }_{4} T \min ^{2}+$ & $a_{5} T \max$ & $+a_{6} U m i d^{2}$ & $+a_{7} T$ max & $T \min +c$ & $a_{8} T \min . U r$ & $d+a_{9} T \mathrm{n}$ & xUmid & \\
\hline$F_{t a b}(C$ & ;10,18) & & & & & & & & & & & \\
\hline
\end{tabular}

mais significativo do que o polinômio sem os termos do segundo grau, $\left(F_{\text {calc }}>F_{\text {tab }}\right)$. Porém, observa-se que a porcentagem da variação explicada, $R^{2}$, indica que o modelo explica aproximadamente $37 \%$ da variação total de W, 25\% da variação total em GS, 1,7\% em PL, 5\% em FN, 2\% em AA e 6\% em EST, para os dados do ano de 2000. Para o ano de 2001, o modelo explica aproximadamente $16 \%$ da variação total em FN, 15\% em AA, 5\% em W, 6\% em PL e 4\% em GU. Pode-se dizer, desta forma, que, apesar de a regressão ser significativa, a relação obtida não é útil para fins preditivos, pois têm-se valores de $R^{2}$ inferiores a 0,70 em todos os resultados de regressão.

\section{CONCLUSÃO}

Nas condições em que os conjuntos de variáveis IQI e IQP foram avaliados, ficou comprovado o relacionamento entre tais conjuntos. O modelo de regressão linear multivariado não se mostrou adequado para descrever o comportamento das variáveis dependentes IQI em função das variáveis independentes IQP; portanto, existem outros fatores que devem ser considerados na explicação da variabilidade do vetor de respostas. Há necessidade de se investigar outras variáveis explicativas e se tentar novo modelo de regressão multivariada. No caso univariado, observou-se que o modelo quadrático é o mais indicado para os dados em questão do que o linear; porém, ainda não é um modelo que possa ser considerado muito bom $\left(\mathrm{R}^{2}<0,70\right)$. Dessa forma, a pesquisa deve prosseguir a fim de se estabelecer o modelo multivariado adequado. 


\section{AGRADECIMENTO}

À gerência da Cooperativa Agrícola de Campo Mourão (COAMO), que gentilmente recebeu os autores e forneceu os dados para esta pesquisa.

\section{REFERÊNCIAS}

BARROS NETO, B. et al. Planejamento e otimização de experimentos. Campinas, SP:UNICAMP, 1995. 299p.

BRUnETTA, D. et al. Cultivares de trigo no Paraná: rendimento, características agronômicas e qualidade industrial. Londrina: EMBRAPA - CNPSo, 1997. 48p. (EMBRAPA-CNPSo. Circular Técnica, 18).

CHAVES NET0, A. Probabilidade e estatística aplicada. Curitiba: UFPR, 1998. 110p. (notas de aula).

GONZALEZ, M.; LOPES, X. Tratamento de farinhas. Santiago, Chile: Granotec, 1992. p.03-24.

GUARIENTI, E.M. Qualidade Industrial do trigo. Passo Fundo: EMBRAPA - CNPT, 1993. 27p. (EMBRAPA CNPT.Documentos, 8)

GUARIEnTI, E.; MIRANDA, M. Métodos de análise recomendados para o programa interlaboratorial de análises de trigo e farinha de trigo. Passo Fundo: EMBRAPA - CNPT, 1999. p.03-18.

GUARIENTI, E.M. Efeito de variáveis meteorológicas na qualidade industrial de trigo. 2001. 240f. Tese
(Doutorado em Engenharia de Alimentos) - Faculdade de Engenharia de Alimentos, Unicamp, São Paulo, 2001.

INSTITUTO AGRONÔMICO DO PARANÁ. Recomendações técnicas para a cultura do trigo no Paraná - 1999. Londrina, 1999. 149p. ilus. (IAPAR.Circular, 106).

INSTITUTO AGRONÔMICO DO PARANÁ. Informações técnicas para a cultura do trigo no Paraná - 2000. Londrina, 2000. 152p. ilus. (IAPAR.Circular, 109).

INSTITUTO AGRONÔMICO DO PARANÁ. Informações técnicas para a cultura do trigo no Paraná - 2001. Londrina, 2001. 174p. (IAPAR.Circular, 116).

JOHNSON, R.; WICHERN, D.W. Applied multivariate statistical analysis. New Jersey: Prentice Hall International, 1988. 642p.

KOEHLER, H. S. Estatística Experimental. Curitiba: UFPR. 1998. P 36-43.

MOOD, A.; GRAYBILL, F. Introduction to the theory of statistics. 3.ed. New York: Mc-Graw-Hill, 1986. 564p.

REUNIÃO DA COMISSÃO SUL-BRASILEIRA DE PESQUISA DE TRIGO, 31. Recomendações da Comissão Sul Brasileira de Pesquisa de Trigo. Passo Fundo: Comissão Sul Brasileira de Pesquisa de Trigo, 1999. 66p.

REUNIÃO DA COMISSÃO SUL-BRASILEIRA DE PESQUISA DE TRIGO, 33. Recomendações da Comissão Sul Brasileira de Pesquisa de Trigo. Passo Fundo: Comissão Sul Brasileira de Pesquisa de Trigo, 2001. 90p. 